Ciência e Natura, Santa Maria v.38 Ed. Especial-

IX Workshop Brasileiro de Micrometeorologia, 2016, p. 467 - 476

Revista do Centro de Ciências Naturais e Exatas - UFSM

ISSN impressa: 0100-8307 ISSN on-line: 2179-460X

\title{
CIÊNCIAPNATURA 35旁
}

\section{Parametrização convectivas e simulação explícita: Testes de sensibilidade para Sistemas Convectivos Amazônicos}

\author{
Convective parametrization and expicity simulation: sensibility tests for Amazonian Convective \\ Systems
}

\section{Vagner Anabor ${ }^{1}$, Franciano S. Puhales ${ }^{1}$, Everson Dal Piva $^{1}$, Otávio C. Acevedo ${ }^{1}$}

Professor Adjunto, Universidade Federa de Santa Maria, Santa Maria, Brasil

\begin{abstract}
Resumo
O modelo WRF (Weather Research and Forecasting Model) foi utilizado para realizar a simulação dos Sistemas Convectivos de Mesoescala ocorridos entre os dias 18 e 21 de junho de 2014 nas proximidades do sítio experiemental ZF-2/LBA, Manaus,Amazonas, Brasil. O objetivo é verificar o impacto das parametrizações convectivas Betts-Miller-Janjic, esquema controlado pela por camada profunda e Kain-Fritsch, esquema controlado por condições de baixos níveis, na simulação de tempestades tropicais Amazônicas. As simulações tem uma duração de $72 \mathrm{~h}$ com domínios aninhados de $12 \mathrm{e} 3 \mathrm{~km}$. A evolução do modo convectivo, outflows e piscinas frias são observados nas duas simulações. O esquema Kain-Fritsch reproduziu tempestades mais realistas com modo convectivo, propagação e downdrafts similares ao usualmente observado.
\end{abstract}

Palavras-chave: Sistemas convectivos tropicais, parametrização convectiva.

\begin{abstract}
The WRF(Weather Research and Forecastiong Model) is used to simulate Mesoscale Convective Systems occurred between 18-21 june 2014 close to ZF-2/LBA experimental site, Manuas, AM, Brazil. The main objective is to verify the convective parametrization Betts-MillerJanjic, a deep layer controled scheme, and Kain-Fritsch, a low level control scheme in simulations of tropical storms over the Amazon. The simulation least $72 \mathrm{~h}$ with nested domains of 12 and $3 \mathrm{~km}$. The evolution of convective systems, convective mode, cold pools and outdrafts were observed. The convective scheme Kain-Fritsch reproduced realistics storms with convective mode, propagation and downdrafts which resembles the usual observations.
\end{abstract}

Keywords: Tropical Convective Systems, Convective parametrization. 


\section{Introdução}

$\mathrm{Na}$ região tropical do globo os Sistemas Convectivos de Mesoescala Tropicais (SCMt) são responsáveis por dois terços da precipitação total (Nesbit, 2006). A convecção profunda em regiões tropicais não corre de forma aleatória, mas sim de maneira organizada em que tempestades convectivas interagem formando sistemas convectivos de mesoecala (Zipser, 2007, House, 2004). Entender como ocorre a organização de sistemas convectivos multi-escala $(0-100 \mathrm{~km})$ é fundamental para o entendimento do balanço de global e dos fenômenos de escala climática. Tais sistemas produzem, muitas vezes, grandes volumes de precipitação, enchentes, ventos intensos e tornados, danos por granizo e tempestades elétricas. Mas estes não são os únicos fenômenos produzidos pelos SCMt, durante seu desenvolvimento intensos fluxos verticais acendentes (updrafts) e descendentes (downdrafts) promovem transporte e mistura do ar estratosférico para dentro troposfera superior (Frey et al., 2015). Estas trocas promovem a intrusão de gazes presentes na estratosfera, como por exemplo o ozônio, para o interior da troposfera. Se os SCMt produzirem downdrafts suficientemente intensos para produzir velocidades convectivas horizontais de $10 \mathrm{~ms}-1$, então concentrações de ozônio em superfície podem aumentar 10-30ppbv (Grant et al., 2008).

A detecção deste transporte vertical de massa em grande escala demandaria a realização de estudos observacionais com redes de alta densidade que, em uma região ampla como a Amazônia, seria dispendioso e de difícil logística, assim, a modelagem numérica apresenta-se como uma alternativa barata para avaliar os processos físicos envolvidos na convecção profunda.

Capturar e prever a convecção no tempo e espaço corretos é crucial para uma simulação realista que represente a variabilidade dos fenômenos meteorológicos . Neste sentido, o uso de parametrizações convectivas possibilita estabelecer uma série de regras para ativar a convecção em horários e locais com potencial para o desenvolvimento de convecção profunda. Os esquemas de parametrização convectiva estão divididos dois tipos principais: esquemas controlados pela camada profunda e esquemas controlados por processos de baixos níveis . Entre os esquemas de parametrização convectiva controlados pela camada profunda mais populares para estudos da convecção tropical está o Betts-Miller-Janjic (BMJ) (Betts e Miller, 1986; Janjic, 1994). Este esquema é fortemente baseado em observações realizadas em regiões tropicais e foi projetado para representar o estado de quase-equilíbrio na convecção profunda, isto é feito basicamente através da comparação de variáveis atmosférica temperatura, razão de mistura e pressão para encontrar pontos de saturação. Este esquema ajusta a atmosfera para perfis de saturação de referência para regiões tropicais. Assim, é fortemente dependente da umidade em níveis médios não levando em consideração as condições atmosféricas de baixos níveis. BettsMiller (1999) incluiu uma abordagem simplificada para downdrafts nos três últimos níveis do modelo, contudo Stensrud (2007) aponta não haver evidências de que este esquema esteja implementado em algum modelo, bem como não está incluso o transporte devido ao momento.

Os esquemas convectivos controlados por baixos níveis são esquemas em que ativação convectiva é governada por processos físicos como instabilidade, umidade e levantamento é necessário para desenvolver convecção profunda (Johns e Doswell, 1992). O esquema Kain-Fritsch (KF) (Kain e Fritsch, 1994; Kain, 2004) é um exemplo típico de esquema controlado por forçantes de baixos níveis e também de fluxo de massa, neste tipo de abordagem forçantes de pequena escala e de mesoescala superam o a inibição convectiva (CIN) e colocam as parcelas em seu nível de convecção livre, a energia potencial convectiva potencial disponível(CAPE) é usada para atingir consumida durante a ascensão livre de maneira similar ao que acontece na natureza. $\mathrm{O}$ esquema KF leva em conta detalhes dos processos convectivos como entranhamento e desentranhamento do ar do ambiente periférico, a magnitude do downdraft está 
relacionado com a intensidade do updraft e as taxas de resfriamento evaporativo e é iniciado $150-200 \mathrm{mb}$ acima da base da nuvem. Aproximadamente $90 \%$ do CAPE é consumido durante o período em que o KF está ativado. Gallus (1999) mostra que o esquema KF produz tempestades com estrutura e volumes de precipitação mais adequados do que BMJ, mas que eventualmente não estão nos locais corretos dentro da estrutura da tempestade. BMJ resulta em uma simulação na qual a localização das tempestades é adequada mas as tempestades não tem a intensidade correspondente as observações.

No dia 19 de junho de 2014, foram registrados dois SCMt nas proximidades do sitio experimental ZF2, Manaus, Amazonas, Brasil. Este evento produziu perturbações no no campo de escoamento e registros de ozônio que indicam um fluxo vertical de massa transportando ar estratosférico em direção a superfície (José Fuentes, comunicação pessoal). Logo, o objetivo deste estudo é verificar o desempenho dos esquemas KF e BMJ na produção de SCMt realistas capazes de produzirem tais perturbações. Serão avalizadas a evolução do modo convectivo, volumes e taxa de precipitação bem como também, a presença e intensidade dos outflows e psicinas frias em simulações explícitas com resolução horizontal de $3 \mathrm{~km}$.

\section{Materiais e Metodologia}

Utiliza-se o modelo Weather Research and Forecastiong, versão 3.6 (WRF). As condições de iniciais advém nálise final (FNL) do modelo global do NCEP com resolução de 1ox1o grau (DOC/NOAA/NWS/NCEP 2000), as condições de fronteira lateral são atualizadas a cada $6 \mathrm{~h}$. A configuração de grades utilizada são apresentadas na fig.1.

A grade mãe possui resolução horizontal de $12 \mathrm{~km}$ (G1) e uma grade foi aninhada resolução horizontal de $3 \mathrm{~km}(\mathrm{G} 2)$, usando-se um esquema two-way-nested (troca de informação entre as grades) e são utilizados 50 níveis verticais em ambos os domínios estão centrados na ZF2 (-2.609097222 S, Long:$60.20929722 W$ ). Os experimentos numéricos são realizados para testar os desempenho das parametrizações convectivas BMJ e KF como condição de fronteira para solução explícita de resolução horizontal de $3 \mathrm{~km}$, nesta escala a parametrização cumulus está desligada. As seguintes parametrizações físicas básicas são: esquema de microfísica WRF de momento simples 3-classes (Hong et al., 2004), o esquema de camada superficial baseado na teoria de similaridade de Monin-Obukov (Monin and Obukhov, 1954), esquema de camada limite da Universidade de Yonsei (YSU, Noh et al. 2003), modelo de transferência radiativa e o esquema para radiação de onda curta Dudhia (Dudhia 1989). Duas parametrizações convectivas foram testadas: O esquema físico de fluxo de massa controlado por baixos níveis: Kain-Fritsch (KF; Kain and Fritsch 1993); - O esquema controlado por camada profunda: Betts and Miller(1986), transofrmado por Janjic' (1994; BMJ);

As parametrizações convectivas estiveram ativas na grade de $12 \mathrm{~km}$ e desligadas na grade de $3 \mathrm{~km}$ para que houvesse resolução explícita da convecção. A simulação dura $72 \mathrm{~h}(00 \mathrm{Z}$ do dia18 até $00 z$ do dia 21/06/2014), o dia 18 foi descartado como spinoff.

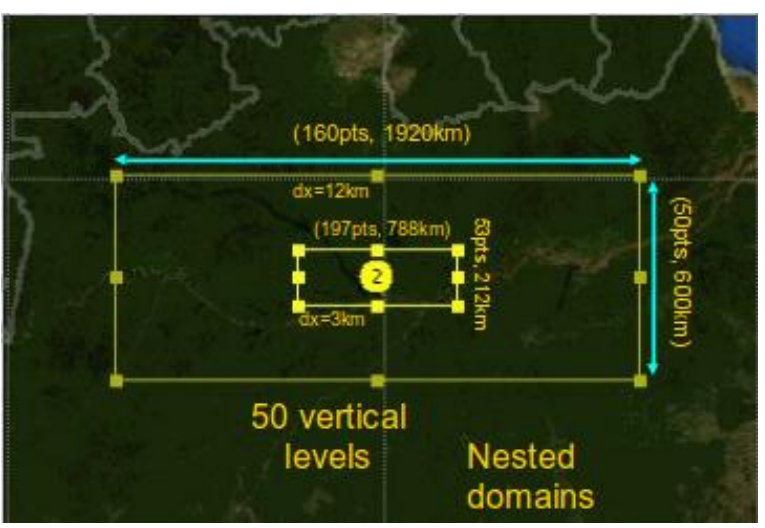

Figura 1: Configuração dos domínios utilizados. 


\section{Resultados}

\subsection{Imagens de Satélite}

As imagens do satélite GOES13,canal IR mostram que, entre os dias 18 e 21 de junho de 2014 (fig.2), sistemas convectivos de mesoescala se formam dentro da bacia Amazônica, com propagação principal para oeste sobre a área da ZF2(proximidades de Manaus). A máxima atividade convectiva na região segue o ciclo diurno da região e ocorre entre o final da tarde e o início da noite. No dias 19 e 20 são observados sistemas convectivos de escala meso- $\beta(\sim 200 \mathrm{~km})$ sobre ou próximos da região de Manaus.

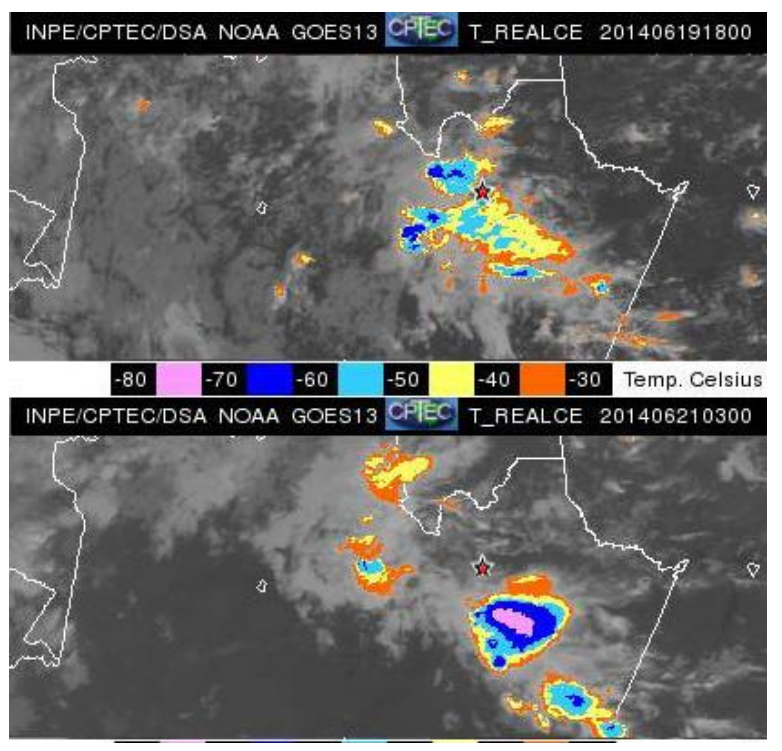

Figura 2: Sistemas convectivos de Mesoescala na ZF2.Temperatura de brilho $\left({ }^{\circ} \mathrm{C}\right)$.

\subsection{Análise qualitativa das simulações}

Reproduzir SCMt através de simulações numéricas representativas quanto sua intensidade, espaço e tempo pode ser particularmente difícil, principalmente em na região Amazônica, onde tem-se escassas observações de superfície e de ar superior. Logo o foco desta análise será o modo convectivo, piscinas de ar frio, padrões no escoamento, padrões de refletividade, tamanho, orientação, tempo de vida e propagação.

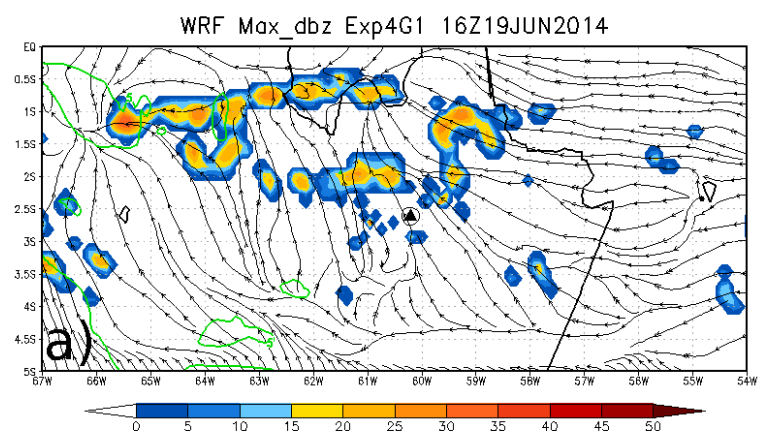

WRF Max_dbz Exp3G1 18Z19JUN2014

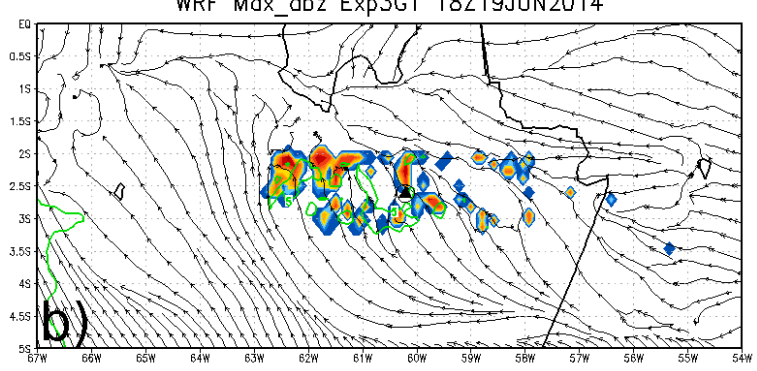

Figura 3: Iniciação e organização dos SCMts, grade de $12 \mathrm{~km}, \mathrm{BMJ}$ (Exp4G1), KF(Exp3G1). Fluxo em superfície (linhas de corrente), magnitude do vento máximo na CLP(linha verde), triangulo ZF2 .

\subsubsection{Iniciação convectiva e evolução dos SCMt}

No dia 19/06/2015 o esquema BMJ (esquema de camada profunda), por ser governado pelas condições de saturação em níveis médios, inicia sua atividade convectiva em G1 próximo das 14:30UTC, organizando alguns núcleos convectivos isolados ao norte da ZF2(fig.4). 


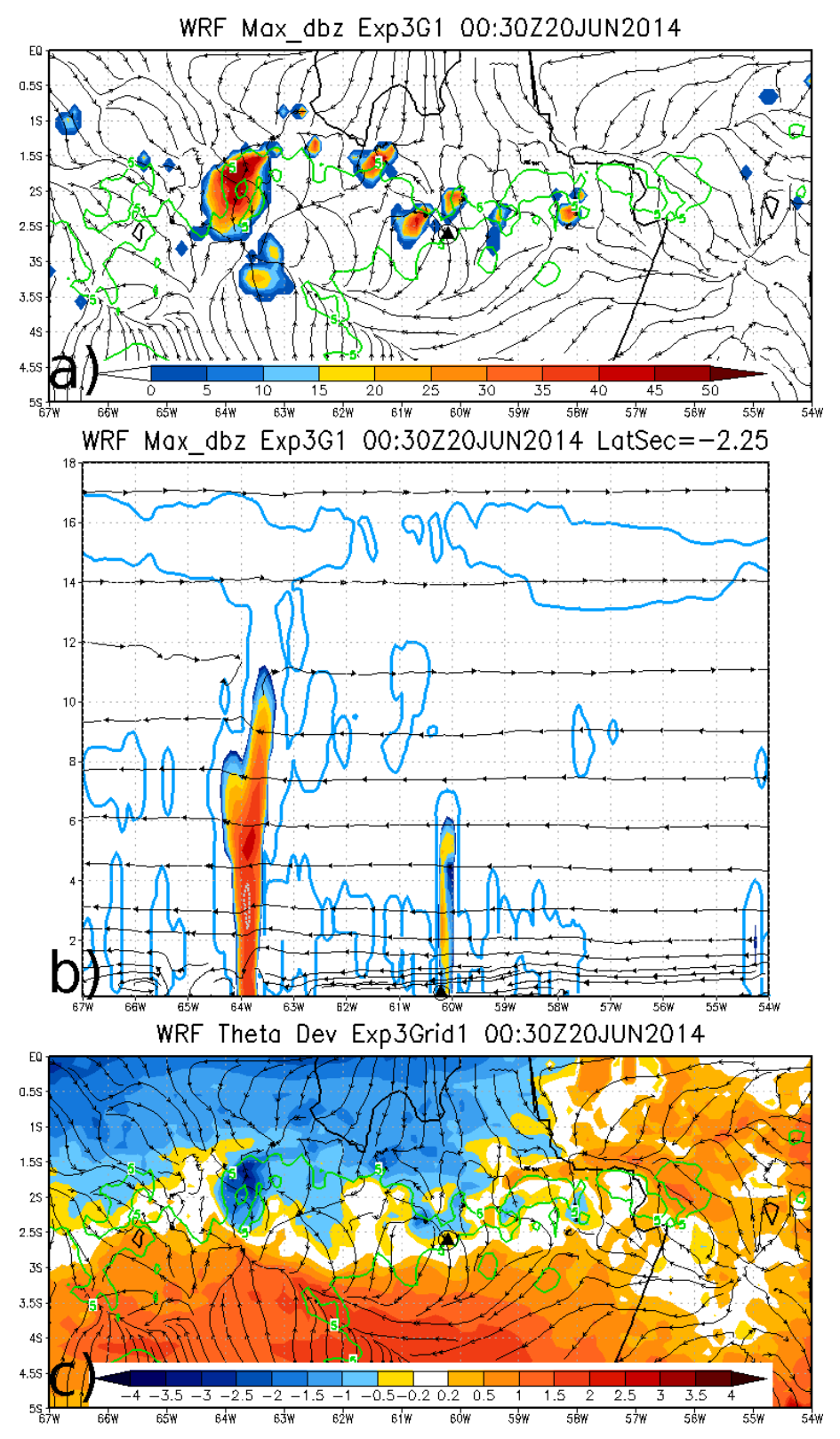

Figura 4: Iniciação e organização dos SCMts, grade de 12km, BMJ (Exp4G1), KF(Exp3G1). Fluxo em superfície (linhas de corrente), magnitude do vento máximo na CLP(linha verde), triangulo ZF2 .

No decorrer da tarde ( 18UTC) estes núcleos tornam-se organizados como SCMt, mas diferentemente do observado nas imagens de satélite, os núcleos convectivos gerados pelo esquema BMJ apresentam propagação na direção sudoeste, mostrando pouca interação com cisalhamento em baixos níveis. Já o esquema o KF, fortemente dependente dos processos físicos de baixos níveis e do crescimento da camada limite para que a convecção seja ativada, gera os primeiros núcleos convectivos aproximadamente 15 UTC e quase que exclusivamente na região correspondente ao interior do domínio de $3 \mathrm{~km}$ (fig.4). Após os primeiros downdrafts os núcleos convectivos se organizam como SCMt. 


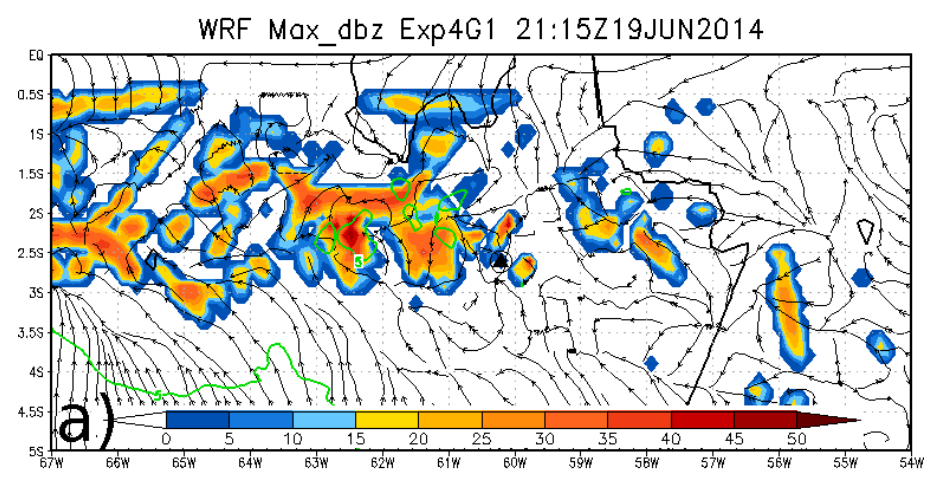

WRF Max_dbz Exp4G1 21:15Z19JUN2014 LatSec $=-2.25$

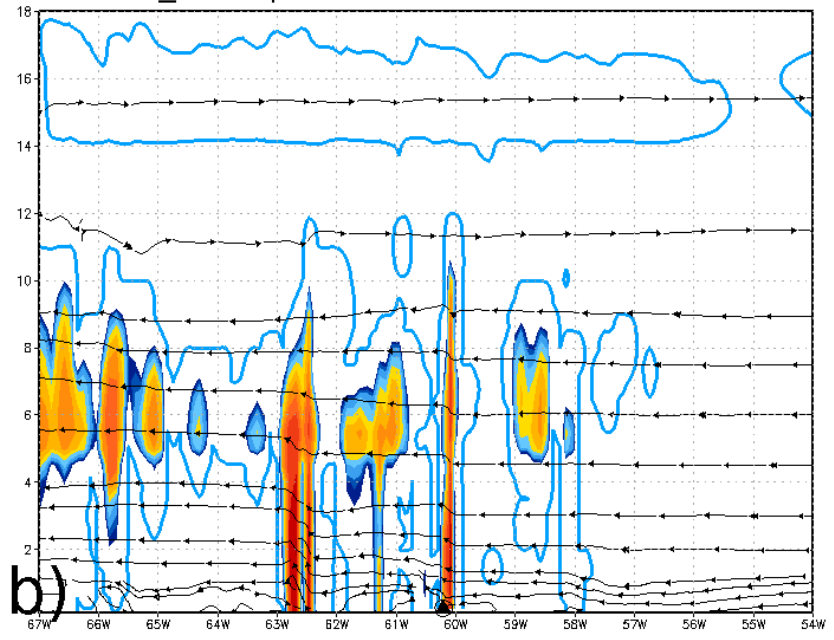

WRF Theto Dev Exp4Grid1 21:15Z19JUN2014

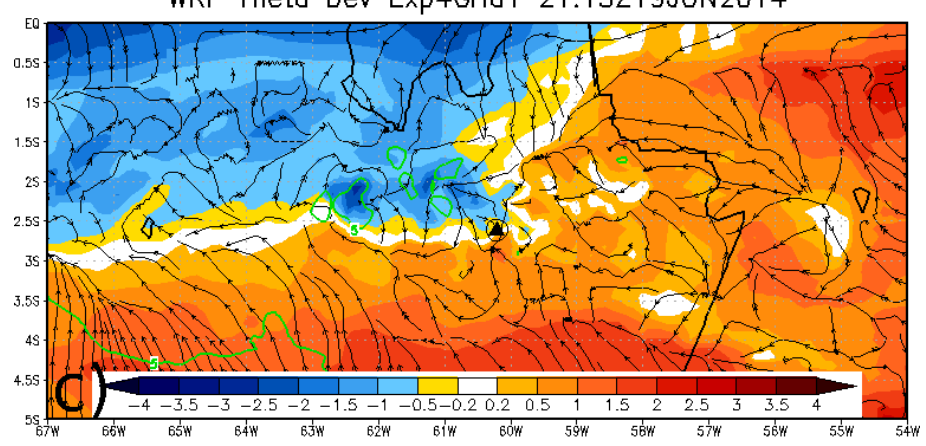

Figura 5: Esquema KF(a)refletividade máxima SCMt maduros e organizados, (b)razão de mistura de nuvem (linha azul), refletividade (dbz, sombreado, escoamento(linhas de corrente), corrente descendente -1 ms-1(pontilhado cinza), (c)perturbação tá temperatura potencial na CLP (sombreado, K), triangulo ZF2

$\mathrm{Na}$ noite do dia 20(figura 5a), já existem SCMt organizados pelo esquema KF conseguem perdurar através da produção de piscinas de ar frio sua interação com o cisalhamento na CLP. Em ambos os esquemas, ocorre há uma significativa redução no aparecimento de novas células convectivas. Weisman et al.(2008) mostra o esquema YSU produzindo em geral CLP mais profundas e secas no Estados Unidos. Os resultados evidenciam o grande impacto destas parametrizações na iniciação e desenvolvimento convectivo. O esquema YSU, que força o escoamento noturno para um regime aproximadamente laminar, inibindo grande parte do desenvolvimento convectivo. 
O esquema YSU, que força o escoamento noturno para um regime aproximadamente laminar, inibindo grande parte do desenvolvimento convectivo. A figura 4 mostra o desenvolvimento vertical foi otimizado pelo esquema KF, conhecido também como esquema de fluxo de massa. Em média as tempestades atingiram $16 \mathrm{~km}$ com a presença de valores de refletividade simulada $>15 \mathrm{dBz}$ até $11 \mathrm{~km}$.

A medida que núcleos convectivos se mesclam formando SCMts maduros esperase observar nas simulações numéricas a presença de downdrafts, padrão divergente no escoamento em baixos níveis e a formação de piscinas de ar frio. Na grade de $12 \mathrm{~km}$, o esquema KF evidencia estes processos apresentando downdrafts $(\sim 1 \mathrm{~ms})$ e outflows $(10 \mathrm{~ms})$ e piscinas de ar $\operatorname{frio}(\theta<-$ $3,5 \mathrm{~K}) \mathrm{da}$ ordem de $10 \mathrm{~ms}$, mas no esquema BMJ não.

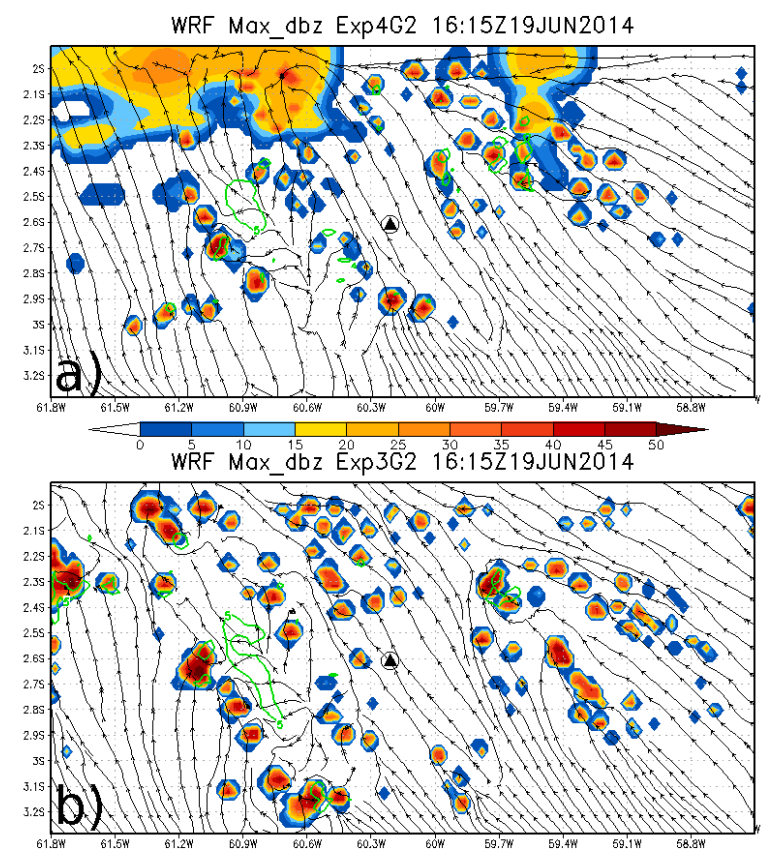

Figura 6: Esquema KF(a)refletividade máxima SCMt maduros e organizados, (b)razão de mistura de nuvem (linha azul), refletividade (dbz, sombreado, escoamento(linhas de corrente), corrente descendente -1 ms-1(pontilhado cinza), (c)perturbação tá temperatura potencial na CLP (sombreado, K)triangulo ZF2

Stensrud (2007) classifica o esquema BMJ como um esquema de camada profunda por não avaliar nem considerar quaisquer condições nos níveis mais baixos da atmosfera, também aponta não haverem evidências de que a produção de downdrafts esteja ativa para esta parametrização cumulus em qualquer modelo existente.

No esquema BMJ(fig.5a), SCMts organizados aparecem rapidamente e podem ser observados ao final do dia 19. Contudo demoram a produzir piscinas de ar frio e frentes de rajadas típicas, indicando que o resfriamento do ar na CPL pode estar relacionado a produção de precipitação pelo modelo (fig.6c). Neste esquema a energia convectiva é consumida quase que instantaneamente a estrutura da convecção apresentada pelo campo de refletividade raramente ultrapassou a altura de $10 \mathrm{~km}$ (fig. 6b). Nas grades aninhadas com resolução de $3 \mathrm{~km}$ (fig.7) ambos os esquemas apresentaram as características típicas observacionais, pois as parametrizações serviram apenas como condição inicial e de contorno para a a solução explicita. As soluções divergem no modo convectivo, evolução e propagação apresentam estruturas coerentes com as presentes nos SCMts.

A iniciação convectiva ocorre aproximadamente ao mesmo temo em ambas as grades mas a convecção originada pelo esquema KF aparece devido aos mecanismos de disparo convectivo e os efeitos do crescimento da CLP enquanto no esquema BMJ a convecção e ativada após a penetração de SCMts pré existentes na grade mãe(fig. 7).

No esquema KFG2, o disparo convectivo é fortemente influenciado pelos processo físicos da CLP. Como ocorre a formação de SCMts organizados na grade mãe, rapidamente as estruturas em G2 tornam-se organizadas. $\mathrm{Na}$ figura $7 \mathrm{a}$ é possível ver núcleos convectivos organizados como um SCMt multicelular, os valores de refletividade mostram que a convecção tem profundidade superior a $10 \mathrm{~km}$ e fluxo vertical de massa promovendo entranhamento em níveis superiores e a produção de downdrafts em ( -1ms-1) em baixos níveis.

Os downdrafts estimulam a formação de piscinas de ar frio que interagem com o escoamento em baixos níveis promovendo o disparo de novas células convectivas na vanguarda do SCMt (fig. $8 \mathrm{~b}$ e $8 \mathrm{c}$ ). O esquema BMJ também é capaz de produzi convecção organizada quando usado para alimentar a solução explícita (fig.9), contudo a convecção é mais rasa raramente atingindo os $10 \mathrm{~km}$ de altura, e formada por SCMt individuais que aparentemente possuem ciclo de vida próprio. 

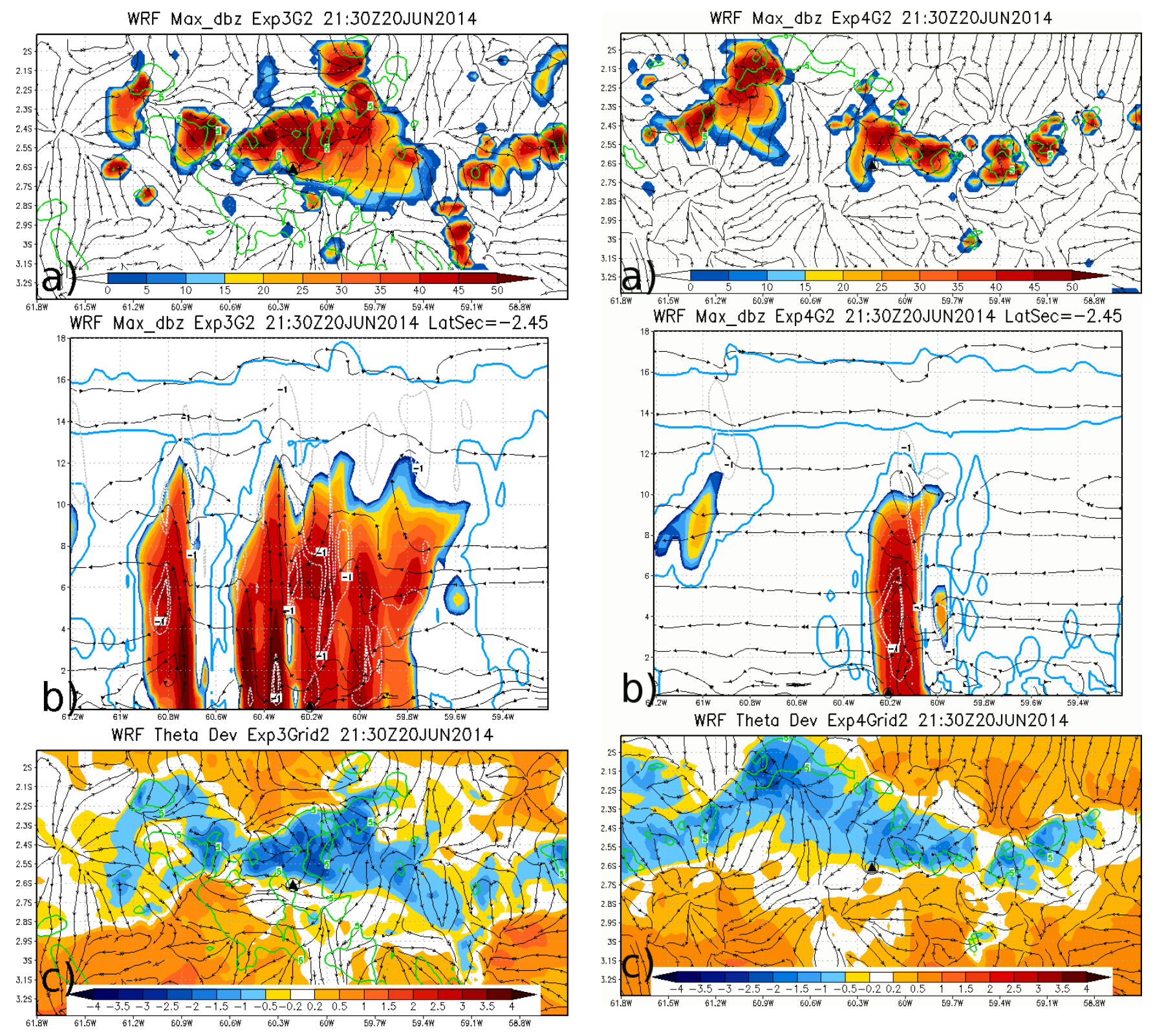

Figura 7: Iniciação e organização dos SCMts, grade de $12 \mathrm{~km}, \mathrm{BMJ}$ (Exp4G1), KF(Exp3G1). Fluxo em superfície (linhas de corrente), magnitude do vento máximo na CLP(linha verde), triangulo ZF2.

Figura 8: Esquema KF(a)refletividade máxima SCMt maduros e organizados, (b)razão de mistura de nuvem (linha azul), refletividade ( $\mathrm{dbz}$, sombreado, escoamento(linhas de corrente), corrente descendente 1ms-1(pontilhado cinza), (c)perturbação tá temperatura potencial na CLP (sombreado, K), ZF2(triangulo) 

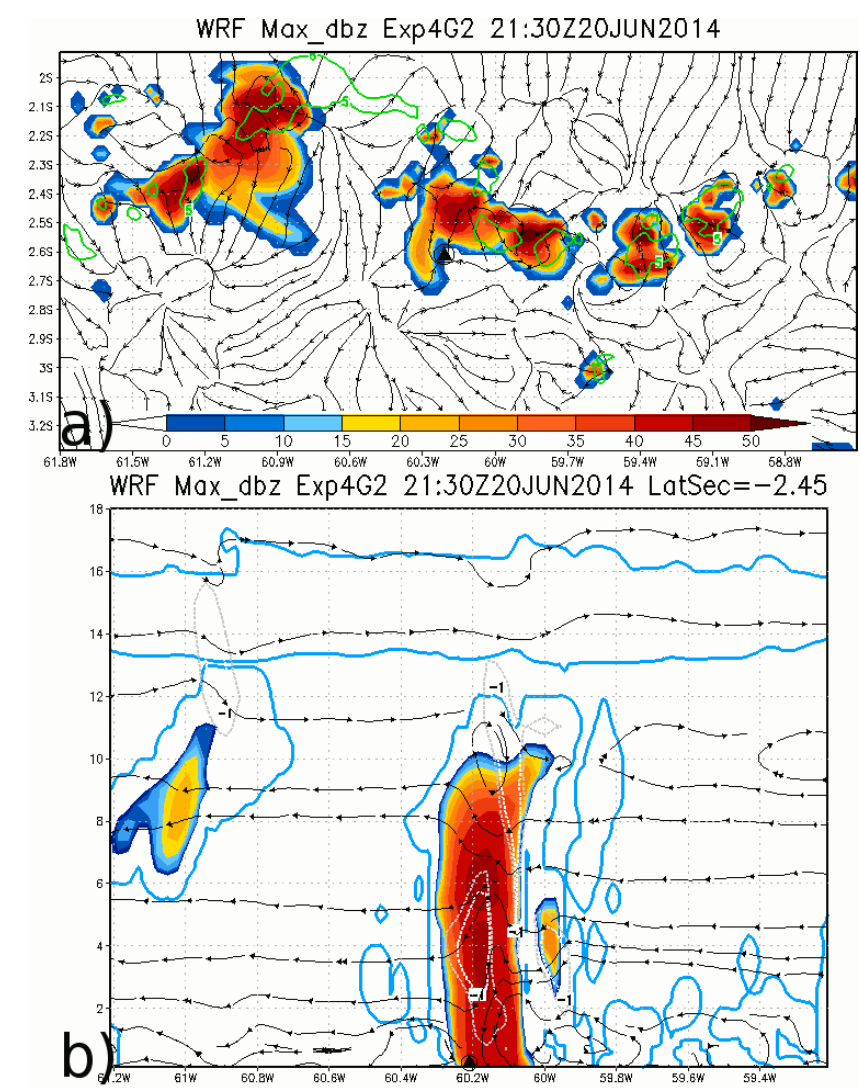

WRF Theta Dev Exp4Grid2 21:30Z20JUN2014

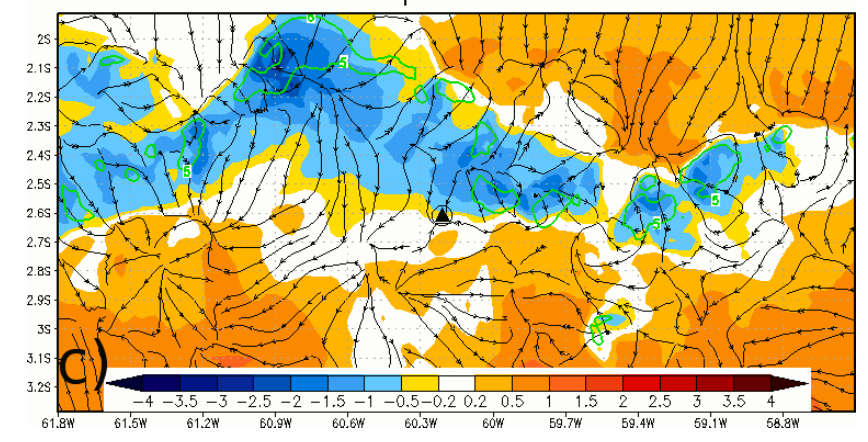

Figura 9: Esquema KF(a)refletividade máxima SCMt maduros e organizados, (b)razão de mistura de nuvem (linha azul), refletividade (dbz, sombreado, escoamento(linhas de corrente), corrente descendente -1ms-1(pontilhado cinza), (c)perturbação tá temperatura potencial na CLP (sombreado, K), ZF2(triangulo). 


\section{Conclusões}

Os resultados mostram que ambos os esquema BMJ e KF foram eficients em produzir produziram convecção proximidades da ZF2. Contudo, nenhum dos esquemas reproduziu com fidelidade a tempestade observada nas imagens de satélite. O esquema KF produziu tempestades mais realista as quais apresentaram o modo convectivo, propagação, downdrafts e piscinas de ar frio mais próximas das comumente observadas na natureza. Por ser um esquema controlado pelos processos físicos de baixos níveis e também por levar em conta o fluxo vertical de massa, é o esquema mais indicado para a reprodução deste tipo de tempestades. Experimentos complementares serão necessários para encontra um esquema de camada limite que consiga manter a atividade convectiva mesmo no período noturno. A má represetação do uso e cobertura do solo e as escassas observações meteorológicas também representam um desafio para este tipo de simulação.

\section{Agradecimentos}

A o Dr. José Fuentes pelas dicussões.

\section{Referências}

Betts, A. K., 1986: A new convective adjustment Part I: Observational and theoretical basis. Quart. J. Roy. Meteor. Soc., 112, 677-691.

Betts, A. K., and M. J. Miller, 1986: A new convective adjustment scheme.Part II: Single column tests using GATE wave, BOMEX, ATEX and Arctic air-mass data sets. Quart. J. Roy. Meteor. Soc., 112,693- 709.
Emanuel, K. A., and M. Z Zivkovic'-Rothman, 1999: Development and evaluation of a convection scheme for use in climate models. J.Atmos. Sci., 56, 1766-1782.

Hong, Song-You, Jimy Dudhia, and Shu-Hua Chen, 2004: A revised approach to ice microphysical processes for the bulk parameterization of clouds and precipitation. Mon. Wea. Rev., 132, 103- 120.

Janjic', Z. I., 1994: The step-mountain eta coordinate model: Further developments of the convection, viscous sublayer, and turbulence closure schemes. Mon. Wea. Rev., $122,927-945$.

Kain, J. S., 2004: The Kain-Fritsch convective parameterization: An update. J. Appl. Meteor., $43,170-181$.

- -, and J. M. Fritsch, 1993: Convective parameterization for mesoscale models: The Kain-Fritsch s:cheme. The Representation of Cumulus Convection in Numerical Models, Meteor. Monogr., No. 46, Amer. Meteor. Soc., 165-170.

Nesbitt, S. W., R. Cifelli, and S. A. Rutledge, 2006: Storm morphology and rainfall characteristics of TRMM precipitation features. Mon. Wea. Rev., 134, 2702-2721.

Stensrud, D. J.: Parameterization Schemes: Keys to Understanding Numerical Weather Prediction Models, Cambridge University Press, New York, 459 pp., 2007.

Johns, R. H. and C. A. Doswell III (1992). Severe local storms forecasting. Wea. Forecasting,7588-612. 